\title{
In vivo retinal imaging in translational regenerative research
}

\author{
Ifat Sher $^{1,2 \#}$, Daniel Moverman ${ }^{1 \#}$, Hadas Ketter-Katz ${ }^{1,2}$, Elad Moisseiev $^{2,3}$, Ygal Rotenstreich $^{1,2 \wedge}$ \\ ${ }^{1}$ Goldschleger Eye Institute, Sheba Medical Center, Tel-Hashomer, Israel; ${ }^{2}$ Sackler Faculty of Medicine, Tel Aviv University, Tel Aviv, Israel; \\ ${ }^{3}$ Department of Ophthalmology, Meir Medical Center, Kfar Saba, Israel \\ Contributions: (I) Conception and design: I Sher, D Moverman, Y Rotenstreich; (II) Administrative support: None; (III) Provision of study materials \\ or patients: None; (IV) Collection and assembly of data: None; (V) Data analysis and interpretation: None; (VI) Manuscript writing: All authors; (VII) \\ Final approval of manuscript: All authors. \\ \#These authors contributed equally to this work. \\ Correspondence to: Ygal Rotenstreich. Goldschleger Eye Institute, Sheba Medical Center, Tel Hashomer 52621, Israel. \\ Email: Ygal.Rotenstreich@sheba.health.gov.il.
}

\begin{abstract}
Regenerative translational studies must include a longitudinal assessment of the changes in retinal structure and function that occur as part of the natural history of the disease and those that result from the studied intervention. Traditionally, retinal structural changes have been evaluated by histological analysis which necessitates sacrificing the animals. In this review, we describe key imaging approaches such as fundus imaging, optical coherence tomography (OCT), OCT-angiography, adaptive optics (AO), and confocal scanning laser ophthalmoscopy (cSLO) that enable noninvasive, non-contact, and fast in vivo imaging of the posterior segment. These imaging technologies substantially reduce the number of animals needed and enable progression analysis and longitudinal follow-up in individual animals for accurate assessment of disease natural history, effects of interventions and acute changes. We also describe the benefits and limitations of each technology, as well as outline possible future directions that can be taken in translational retinal imaging studies.
\end{abstract}

Keywords: Retinal imaging; optical coherence tomography (OCT); fundus imaging; adaptive optics (AO)

Submitted May 31, 2020. Accepted for publication Aug 05, 2020.

doi: $10.21037 /$ atm-20-4355

View this article at: http://dx.doi.org/10.21037/atm-20-4355

\section{Introduction}

The retina is a leading target tissue for advanced therapeutic approaches due to the compartmentalized nature of the eye, its relative immune privilege, and low dose requirement for treatment. Numerous translational studies in recent decades have assessed the regenerative potential of stem cells and gene therapy, which have led to promising clinical trials and the recent approval of the first gene therapy for treating retinal degeneration by the US Food and Drug Administration (1).

Numerous studies demonstrated a direct correlation between retinal structure and visual function in animals and humans (2-7). Traditionally, retinal structure was determined in translational regenerative studies by histological analysis which required sacrificing the animals, removing the eyes for sectioning, hematoxylin and eosin staining, quantification of retinal layer thickness, and immunofluorescence staining for specific cell markers or ultrastructural analysis of the specific retinal cells by electron microscopy (3,8-23). Histological analysis requires a large number of animals, does not enable longitudinal follow-up, easily permits transient changes to be missed, and most importantly cannot be employed in human clinical trials.

$\wedge$ ORCID: 0000-0003-2366-1779. 
The normal transparency of the anterior segment and lens enable relatively easy imaging of the posterior segment. The breakthroughs in advanced imaging techniques enable non-invasive, non-contact, and fast monitoring of changes in retinal structural in vivo without necessitating sacrificing the animals. Thus, they enable monitoring of individual animals in longitudinal studies, can detect transient structural changes, and require a smaller number of animals which reduces both variability and cost. Given that the majority of these imaging modalities are used currently in clinical practice to evaluate human retina, the results of these animal studies using in vivo retinal imaging may be directly translated into clinical trials. Within the last two decades the use of ocular imaging in animal model studies has expanded. Currently, they are gradually being used along with histological analysis for characterizing the natural history of the disease, monitoring the distribution of injected therapeutics, and for assessing their effects on retinal structure and function, as detailed in this review.

Current technology allows for ophthalmologists to capture in vivo images of the retina at a wide variety of resolution. Macroscopically, it permits capturing images of the entire retina [e.g., ultra-wide field fundus imaging $(24,25)$ ] down to a single diseased cell [e.g., using adaptive optics (AO) (26)]. Other innovative single cell resolution imaging technologies include the identification of single apoptotic retinal ganglion cells (RGCs) [using the recently developed Detection of Apoptosing Retinal Cells (DARC) which is currently being considered for use in clinical trials], as well as in vivo determination of the function of single cells by calcium-imaging $(27,28)$. Several of these imaging technologies were adopted from the clinical setting to usage in animal models [e.g., optical coherence tomography, OCT (29)], with others gradually transferring into clinical trials after being developed and validated in animal models (e.g., DARC). In this review, we focused on leading ophthalmic imaging modalities that are being used in today's translational studies, then describe their use and key advantages and limitations associated with each technique.

\section{Color fundus photography}

After the advent of photography's introduction in the mid 1800's, the field of ophthalmology has progressed significantly in its ability to better detect, identify, and observe different ocular pathologies that weren't before possible with the naked eye. Yet with ever advancing photographic technologies provided in today's era, the principle of basic fundus photography remains a cornerstone in ophthalmic imaging. Color fundus photography allows the assessment of the gross morphology of the retina in vivo. Furthermore, it allows for identification of structural changes in a wide range of ocular pathologies from diabetic retinopathy to the rare Oguchi disease $(30,31)$. It can be used in humans and animals, both large and small, and is a key tool for identification of lesions, geographic thinning, subretinal deposits, evaluation of retinal vasculature, and the optic nerve head. In translational studies, color fundus imaging is widely used for the characterization of new animal models, determination of the natural history of the disease and staging (32-35), as well as for monitoring the location and distribution of injected therapy. Specifically, a vast majority of advanced therapies, including stem cells and gene therapy, are delivered into the posterior segment by a subretinal injection, and the resulting bleb of elevated retina following subretinal injection and the eventual resolution can be visualized by color fundus photography (36-39). In recent studies involving subretinal transplantation of whole retina grafts or retinal pigment epithelial cells grown on scaffolds, fundus imaging was used for evaluating graft location and health of the surrounding tissue $(39,40)$. Fundus imaging was also used as a gross qualitative estimation of the safety of intraocular injection of viral vectors (41) or cells and for evaluating the potential regenerative effects of various therapies on the retina (42). The main limitations of fundus imaging are the lack of details (resolution and retinal layer), the lack of quantitative measurements, and the subjective interpretation of the results. Therefore it is almost always just one part of the ophthalmic evaluation, often accompanied by histological or OCT analysis (41). Recent artificial intelligence approaches are being developed for objective, standardized tagging of fundus images (43), however these have not been employed yet in animal studies.

\section{Near infra-red fundus imaging}

One advancement of fundus imaging was the development of near infra-red (NIR) fundus imaging. It involves capturing the fundus autofluorescence (FAF) by excitation at $787 \mathrm{~nm}$ and capturing the emission at $>800 \mathrm{~nm}$. Using a longer wavelength for excitation is advantageous because it enables the detection of sub-retinal features, both normal and pathological, by penetrating deeper through the fundus as compared to other modalities. Retinal imaging using 

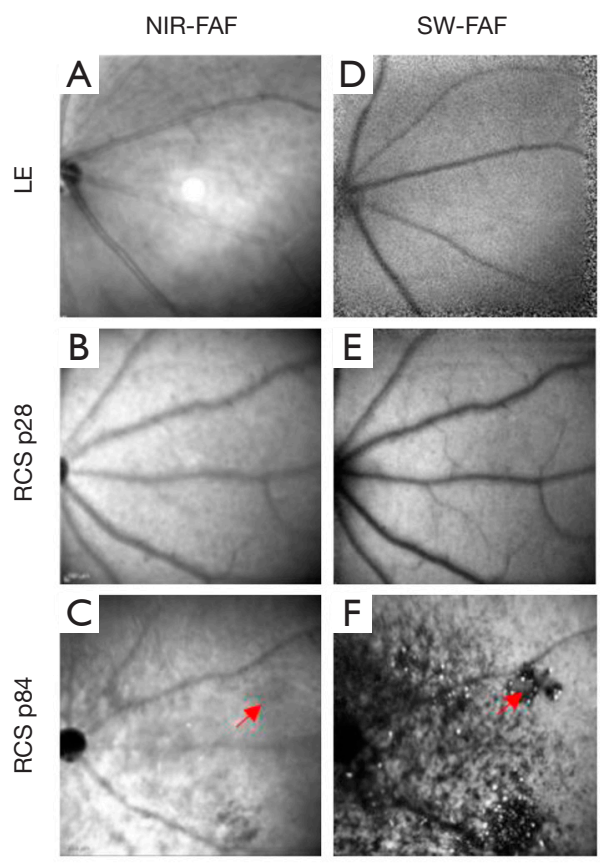

Figure 1 NIR-FAF $(A, B, C)$ and SW-FAF $(D, F)$ imaging of WT, non-dystrophic Long Evens (LE) rats (A,D) and dystrophic RCS rats at age postnatal day $[\mathrm{p}] 28(\mathrm{~B}, \mathrm{E})$ and p84 (C,F). At p28, the SW-FAF signal is weak. At p84, the SW-FAF image of RCS rats is characterized by a strong hyperautofluorescence and the appearance of discrete hypofluorescent lesions surrounded by hyperfluorescent flecks around the optic nerve head. No hypofluorescent lesions are found in LE rats. These hypofluorescent lesions are barely detectable by NIR-FAF (C, red arrow points to a dateable lesion). The figure is modified from our previous publication (55).

NIR-FAF was first reported in 1996 by Piccolino et al. and Elsner et al. $(44,45)$. Later studies demonstrated that the source of NIR-FAF is mainly retinal pigment epithelium (RPE) melanin, and to a lower extent, the melanin in choroidal layers (46). Hence, this imaging modality can be used to study the pathophysiology of diseases affecting the RPE, Bruch's membrane and choroid, and for evaluating the effect of interventions on these retinal layers (47). Recent development of ultra-wide field imaging enables monitoring the NIR-AF in a larger area of the retina $\left(102^{\circ}\right)$ than conventional FAF $\left(55^{\circ}\right)$, and is mainly used in translational studies for safety assessment of therapeutic injection, including gene therapy (48). Similar to color fundus imaging, the main drawback of NIR-FAF is the lack of quantitative measures and the subjective interpretation of the results.

\section{Short-wavelength fundus autofluorescence (SW- FAF)}

SW-FAF is another method for gross assessment of RPE health across the retina. It is based on excitation at 488 $\mathrm{nm}$ (therefore known as Blue-FAF) and a subsequent recording of the emitted autofluorescence by lipofuscin using a barrier filter between 500 and $700 \mathrm{~nm}$ to exclude for emittance from other retinal pigments (49). Lipofuscin pigments accumulate in RPE cells (50) and are comprised of a mixture of fluorophores produced in the disk membranes of photoreceptor outer segments (POS) as oxidative byproducts of vitamin A use and recycling. The pigments are transferred to RPE cells after the cells phagocytose the shed POS (51,52). Ex vivo the peak excitation of lipofuscin is between $450-490 \mathrm{~nm}$, and the maximal emission is at $\sim 600 \mathrm{~nm}$ (53). In the healthy retina, the SW-FAF increases with age with the continuous deposition of Lipofuscin in the lysosomal RPE cells (54). In areas with pathological loss of RPE and/or photoreceptor cells, both the SW-FAF and the NIR-FAF signals are significantly attenuated or lost, but SW-FAF imaging is more sensitive for these pathological changes, as demonstrated in Figure 1 (55-57). As fundus imaging is noncontact, noninvasive and fast, it is highly valuable in the detection and longitudinal monitoring of geographic atrophic lesions. The development of tools providing automated quantification of hypo-fluorescent lesion sizes has led the way to employing this imaging tool as an endpoint in clinical trials with GA patients $(4,58,59)$. Unfortunately, these automated quantification tools are not directly applicable to image small rodent eyes. Recently, a readily available semi-automatic tool for quantification of hypofluorescent lesions in rodent eyes was developed based on the "Regional Finder" tool available in the Heidelberg Engineering OCT systems (55). This tool was successfully employed for monitoring retinal degeneration in Royal College of Surgeon (RCS) rats, a widely used model in translational studies for regenerative medicine. In this rat model, loss-of-function of the MERTK gene prevents POS phagocytosis by the RPE cells. The shed discs accumulate in a subretinal layer termed the "debris zone", leading to an increased SW-FAF hyperfluorescence. This is shortly followed by a gradual replacement of the hyperfluorescent lesions with hypofluorescent lesions due to a loss of photoreceptors and shed debris, as demonstrated in Figures 1,2 (55). The benefit of using SW-FAF is its ability to monitor retinal degeneration in rats throughout the entire retina using just a few scans, which is practically 
SW-FAF
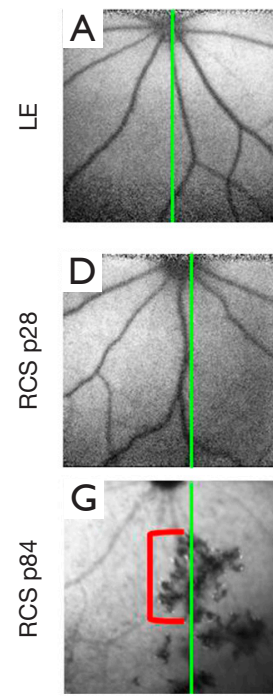

SD-OCT
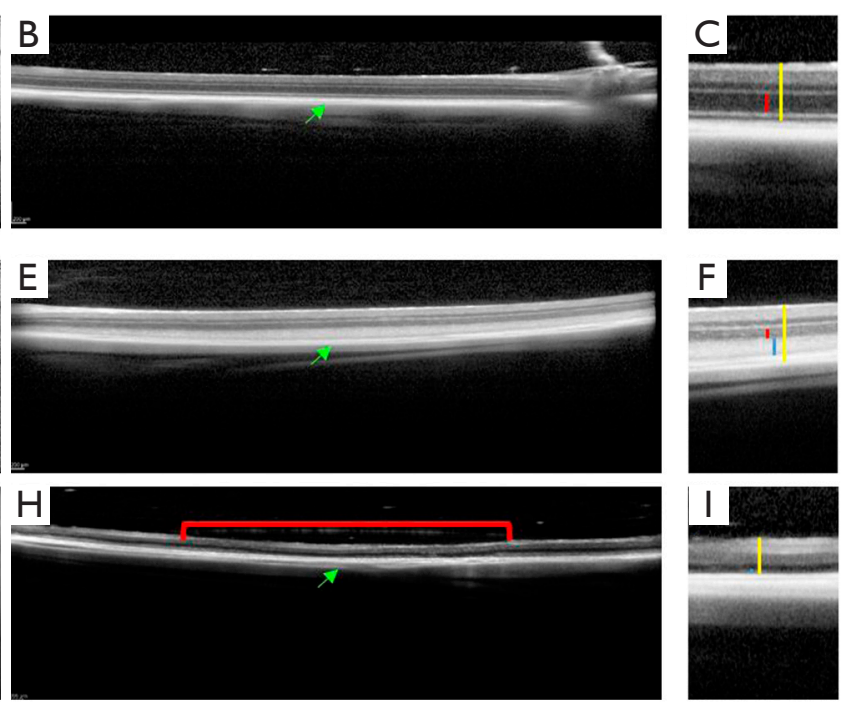

Figure 2 Multimodal imaging for longitudinal follow-up of retinal degeneration in RCS rats. SW-FAF imaging (A,D,G) and corresponding SD-OCT scans $(\mathrm{B}, \mathrm{E}, \mathrm{H})$ of the central retina area in a representative WT, non-dystrophic Long Evans (LE) rat and a single-representative RCS rat at different ages (p28, p84). (C,F,I) A zoomed-in view of the SD-OCT shown in (B,E,H), respectively. The vertical lines in panels (C,F,I) highlight the ONL (red), total retina (yellow) and debris zone [DZ, in blue, panel (F)]. The hypofluorescent lesion detected in the SW-FAF scan [highlighted by red lines, (G)] corresponded to focal loss of the DZ (H,I). The green vertical lines in panels (A,D,G) indicate the position from which the SD-OCT scans on the right were taken.

impossible to do using other imaging techniques, such as OCT, as detailed below.

SW-FAF can further be employed in longitudinal followup studies of retinal degeneration and in mouse models of lysosomal storage diseases where it is possible to visualize accumulating autofluorescent storage biomolecules in the inner retina (60).

In addition to using SW-FAF in natural history translational studies, since SW-FAF can detect changes in subretinal structures, it is used in translational studies for assessing the safety of subretinal injection procedures. For example, a recent study using primates demonstrated hypofluorescent areas outside the subretinal injection bleb, correlating with RPE and outer retina structural abnormalities caused by re-administration of subretinal gene therapy viral vectors (37).

\section{Fluorescent fundus imaging}

Preclinical animal studies often use fluorescently labeled cells or proteins (such as green fluorescent protein, GFP) in order to identify and localize the injected therapeutics in the target tissue, and to determine vector expression levels in gene therapy studies specifically at early stages of intervention development. Several animal-specific imaging systems have been developed for fluorescent imaging in small animals (e.g., Phoenix MICRON ${ }^{\mathrm{TM}}$ systems). Moisseiev et al. used in vivo scanning laser ophthalmoscopy (SLO) fundus imaging to visualize enhanced GFP (EGFP)-labeled human bone marrow stem cells within the eyes of a mouse model of retinal degeneration following intravitreal injection (61). Wassmer et al. used fluorescent fundus imaging to compare the efficacy of intravitreal delivery of GFP into the retina using AAV2 vectors and exosome-associated AAV2 (62). The clear advantages of such an approach are the abilities to monitor and compare GFP expression levels at different time points following gene therapy delivery in the same animal, and to assess the fluorescent protein expression levels at various retinal areas (e.g., center of the retina vs. optic nerve head) in a single imaging frame. However, removal of the eyes and processing retinal sections for fluorescent or confocal microscopy are required to determine which retinal layers expresses the protein and to evaluate the temporal changes in gene expression level in each retinal layer (62). 


\section{Confocal scanning laser ophthalmoscopy (cSLO)}

In the 1980's a new technology took advantage of the optical properties of the eye by using a laser to capture the retina at a cellular resolution, in what is today called confocal scanning laser ophthalmoscopy (cSLO) (63). cSLO uses a laser light at a specific wavelength, pinhole apertures arranged in precise confocal positions, and a beam splitter to generate a raster pattern scan of the retina that is subsequently detected and converted into an electrical signal to allow for its reconstruction into a detailed digital image. Similar to other fundus imaging technologies, cSLO enables the evaluation of gross changes in retinal and vascular structures of the posterior segment en-face. Using the lasers at different wavelengths in the shorter range [usually native Argon wavelengths (488/514 nm)], and infrared diode lasers in the longer wavelength range $(795 / 830$ or $785 / 815$ $\mathrm{nm}$ ), enable detection of RPE irregularities and hypo- and hyper-autofluorscent areas that reflect metabolic changes in photoreceptor and RPE cells. It is always coupled with OCT and histological analysis to gain information on the thickness of the retinal layers.

This imaging modality was successfully used for enface visualization of the retina in several mouse and rat models of retinal degeneration (e.g., Rho-/-, rd1, RPE65-/-, $R b o^{P 23 H}$ ), and in characterization of the gross structural changes associated with disease progression $(64,65)$. This imaging modality was also employed in a study aimed at development of sodium iodate induced retinal degeneration in mice. As animals were scanned at different time points following sodium iodate intravenous injection, cSLO revealed the appearance of dark patchy blots in planar images of the retina that corresponded to areas with photoreceptor loss by OCT and histology analysis, and were dose dependent (66). Even though the meticulous comparison between cSLO, OCT, and histological analysis of the animals demonstrated the superiority of OCT in early detection of photoreceptor loss compared with the cSLO en face imaging (64), cSLO remains a valuable tool for longitudinal follow-up covering large areas of the retina since OCT enables imaging of only small areas of the retina. Furthermore, cSLO fundus imaging enables quick scanning of animals at different time points and can assist in choosing the appropriate drug dose, as well as the optimal time points and retinal locations for performing other, more laborious analyses such as OCT scans, histological, and functional assessments.

\section{OCT}

OCT is a noninvasive tomographic imaging technique that provides high-resolution cross sectional two- and threedimensional imaging of microscopic retinal layers based on the principle of low-coherence tomography (67). It does so by comparing the measured backscattered light from a tissue sample, such as the retina, and a known reference arm, such as a mirror, to create an interference pattern that creates an image when the two light paths match or fall within the coherence length of the wave (29). Different technological advancements over the years have risen to the development of time-domain OCT, spectral-domain OCT (SD-OCT), swept-source OCT, and long-wavelength OCT (68).

OCT is widely used in the clinical setting because it provides objective and quantitative measurements of retinal layer thickness, edema, and subretinal retinal fluids/lesions (69). Thinning of retinal layers, specifically photoreceptors layers in retinal and macular degeneration diseases, along with RGC and nerve fiber layers in glaucoma and other optic nerve degeneration diseases, reflect loss of nerve cells and correlate with visual function deficits (5-7,70-72). OCT has been widely used in the last decade for in vivo noninvasive characterization of retinal structure in animal models, reducing the numbers of study animals needed and enabling for assessment of acute dynamic processes such as retinal edema or thinning (73). Specifically, animal studies enable for directly determining the correlation between retinal thinning as observed by OCT imaging with histological analysis of the retina. Thus, the natural history of disease progression was determined by OCT and validated by histological analysis in numerous retinal degeneration models including RCS rats $(57,74,75)$, Rhodopsin $^{P 23 H}$ and Rbodopsin ${ }^{S 34 T}$ rats (65,76-79); sodium iodate-induced retinal degeneration in rats (66); NRL, Crb1, Rb, Rbo, RPE65 knockout and rd1, rd10, rd12, mice $(64,78,80,81)$, rabbits $(82,83)$, and monkeys (84). In addition, OCT imaging enabled retinal structural changes to be demonstrated longitudinally in various animal models of other retinal diseases including mouse models for lysosomal storage diseases (60) and diabetic retinopathy $(85,86)$ as well as retinal ganglion cell (RGC) death that preceded retinal nerve fiber layer (RNFL) thinning in a mouse model of optic nerve crush (87). These natural history studies paved the way for using OCT imaging for evaluating safety and efficacy of novel intervention using 
a fewer number of study animals, as disease progression analysis can be done using serial imaging of the same animal. These include regenerative studies using stem cells $(84,88-90)$, retinal transplantation (91), laser therapy (92), gene therapy (93) and pharmaceutical interventions (83). The ability to correlate animal model findings with clinical findings in patients carrying the same mutation [e.g., (94,95)] facilitates the translation of these interventional studies into clinical trials. In addition, new drug delivery systems for administrating treatments to the posterior segment can be evaluated in small and large animals using OCT (48).

Some published translational studies were able to obtain high resolution OCT images of the retina in animal eyes by using commercially available clinical grade OCT instruments equipped with eye tracking to prevent image signal disturbance from animal breathing. Some of these instruments are also equipped to improve OCT image resolution by using an automated software that rescans the same area of the retina and averages multiple images $(55,96,97)$. However, one of the limitations in using the clinical devices is that the automatic layer segmentation procedures developed for the human eye are not applicable to the small rodent eyes. Hence, many of these studies report manual layer segmentation by the researcher which by nature is subjective, error prone, and laborious. To overcome this limitation, novel tools are being developed for automatic segmentation of rodent OCT images to enhance the accuracy of segmentation and quantification of retinal layer thicknesses (98). In addition, although there is a wide-field OCT that can scan the whole eye, standard OCT machines are not equipped with this technology and thus have a limited field of view $(99,100)$.

\section{OCT angiography (OCTA)}

One of the hallmarks of retinal degeneration is the characteristic of lowered blood flow coupled with constriction and attenuation of retinal blood vessels (101). In addition, retinal vascular diseases, such as diabetic retinopathy, is associated with changes in retinal vasculature. Recently, OCT-angiography (OCTA) technology has emerged that can detect changes in retinal and choroidal circulation noninvasively in human and animal eyes (102-105). Until the development of OCTA, fluorescein angiography and indocyanine green angiography were used for in vivo visualization of retinal and choroidal circulation. However, these angiographic methods are invasive and provide only two-dimensional imaging of the retinal and choroidal circulation (106). With fluorescein angiography, visualization of the choroidal circulation is limited by the overlying RPE. In contrast, OCTA provides a threedimensional image of the retinal and choroidal circulation by detecting the movement of blood cells in rapid sequential OCT imaging of the same region of the retina (107). Thus, OCTA images can be obtained using OCT instrumented equipped with the appropriate software. It is relatively fast and does not require injection of dyes (108). In recent years, a growing number of clinics and animal translational studies have been using this imaging modality (109). Major limitations of OCTA include its large dependence on image interpretation, possible artifacts from eye movements (110) and projection artifacts from overlaying vessels (111), as well as possible segmentation errors that require manual correction (112). In addition, there is no global standardized and reliable segmentation method, leading to variability in segmentation by different users that may limit the ability to compare between studies led by different groups (113). Also, the current OCTA technologies enable only a small imaging area $\left(3-12 \mathrm{~mm}^{2}\right)$ with high resolution (110). Nevertheless, several studies reported the use of OCTA for monitoring longitudinal changes in retinal vasculature in wildtype rodents (114), and in rodent models of retinal degeneration including rd10 mice (115). OCTA has also been used to study the protective effects of novel interventions in rodent models of retinal vasculopathy (116). As more researchers get access to this technology and gain experience in interpreting the data, OCTA may allow for the extension of knowledge on vascular changes associated with retinal disorders and provide a deeper assessment of the effects of new treatments on retinal and choroidal vasculature.

\section{AO}

$\mathrm{AO}$ is a technology originally developed by astrophysicists to remove the atmospheric turbulence present while viewing objects in outer space (26). In recent years, this technology has extended into ophthalmology, where it is used to correct for optical aberrations and allow for direct in vivo visualization of the retina at a cellular level (117). Its complex system composes of three principle componentsa wavefront sensor to measure incoming aberrations, a corrective element, and a software system to control the interplay between the previous two (118). There exists a range of corrective, or "adaptive", elements that allow for the incoming wave properties to be reconfigured as they hit the system such as liquid crystal modulators, digital mirror 
devices, or deformable mirrors (119). AO by itself does not provide a retinal image, but rather is a subsystem that must be incorporated into a pre-existing device such as a fundus camera, a cSLO system, or an OCT system $(26,120)$. $\mathrm{AO}$ technology is an impressive emerging technology that provides unprecedented retinal visualization to as small of a detail as a single photoreceptor cell (121). It produces imaging at a cellular level that is only rivaled by invasive histological procedures (122). For example, when paired with SD-OCT, AO allows for improved photoreceptor visualization on the backdrop of the three-dimensional scaffolding that the OCT system provides (123). In retinal degeneration diseases such as Age Related Macular Degeneration (AMD), AO paired SD-OCT captured clear images of photoreceptor morphology that were congruent with histopathological reports (124). Given AO's ability to map photoreceptor density (125) it has been a sought-after technology to incorporate into animal model studies assessing photoreceptor health, mapping the dynamic interaction between microglia and retinal cells in healthy retina and following photoreceptor injury (126), and for long term monitoring of retinal health following pharmaceutical intervention or cellular therapy (127). The major limitation of $\mathrm{AO}$ is the small area that can be imaged, usually $4 \times 4$ degrees (127). In addition, although the visualization by AO paired technology is impressive, it still remains a question to whether the recovery of photoreceptors in this case concretely translates into visual function (128). In a very recent paper, McGregor et al. combined AO ophthalmoscopy with calcium imaging and successfully recorded RGC activity in the fovea of living primates that received optogenetic treatment after photoreceptor ablation (28). Given the biological and anatomical similarities of the fovea in primates and humans, the potential for this therapeutic approach to translate into clinical studies is encouraging. The applications for $\mathrm{AO}$ integrated imaging are vast but they remain very expensive, require accurate alignment, and need expert monitoring at all times. Although it may be easy to train ophthalmologists to read and interpret the images captured, training individuals to handle and operate the equipment is a significant roadblock to its widespread use (129).

\section{Detection of apoptosing retinal cells (DARC)}

DARC is a promising new technology that utilizes cSLO's detailed image acquisition to detect RGC apoptosis in vivo. Annexin V, a cellular protein which has been demonstrated to bind with high affinity to outer leaflet phosphatidylserine in apoptosing cells (130), is tagged with a fluorescent marker and injected intravitreally. cSLO's set wavelength laser excites the fluorophores and subsequently captures the sensitive fluorescent signals of the dying RGCs (27).

Detection of dying RGCs is decidedly important, as their loss leads to glaucoma, one of the leading causes of irreversible blindness worldwide affecting more than 70 million people $(131,132)$. The gradual visual field loss in glaucoma can go unnoticed at first by the patients, hence early detection of the disease is of significant importance. Using DARC may potentially allow for an early diagnosis of glaucoma, subsequently leading to earlier interventional action and improved treatment outcomes (133). Recently, a push in research to apply DARC to map retinal cell health progression after drug or other pharmacological delivery has been set in motion. Davis et al. reported lower RGC cell death by DARC imaging, which was validated by histological analysis and immunofluorescent staining of Brn3 (an RGC cell marker), following topical administration of coenzyme Q10 and D- $\alpha$-tocopherol polyethylene glycol 1000 succinate in a rat model of ocular hypertension (134). Other studies have employed the DARC technology for detecting apoptotic RGCs in other neurodegenerative diseases such as Parkinson's disease (PD) in mice. In a rotenone-induced model of $\mathrm{PD}$, longitudinal follow-up using DARC demonstrated RGC apoptosis as early as 20 days following rotenone insult, whereas loss of dopaminergic neurons was only found by histologic means in the substantia nigra of the brain on day 60 . The anti-inflammatory drug rosiglitazone prevented loss of RGCs and dopaminergic neurons (135). This study highlights the power of the DARC method for in vivo quantitative monitoring of RGC apoptosis and for screening new pharmaceutical interventions. Further, it highlights the concept of the retina being a "window to the brain" such that these advanced imaging technologies may potentially contribute for earlier diagnosis and screening of new treatments, not only for ocular diseases, but for brain neurodegenerative pathologies as well. Although the technique has demonstrated successes, one challenge it faces is the need for large-scale validation of whether or not DARC is able to effectively distinguish between pathological and age-related physiological RGC death (136). Standardizing the pathological to physiological apoptosis rate of the human eye is the next step before implementing this method in clinical practice (137). Nevertheless, DARC is being explored in clinical trial as a novel method 
for detecting changes in the ganglion cells of the retina associated with glaucoma (NCT02394613).

\section{Conclusions and future aims}

The major benefit of having different in vivo retinal imaging modalities is that they allow the experimenter to select just how macroscopic or microscopic their data needs to be analyzed. For example, capturing an image with the standard fundus camera is the simplest, most convenient, accessible, and most straightforward method of analysis on the market today. The logistics of operating the equipment aren't too different than using a handheld or smartphone camera, as it mostly involves a "point and shoot" method of image capture. Further, the diagnostic power of its seemingly rudimentary macroscopic image can't be overlooked, as not every experiment needs an OCT, $\mathrm{AO}$, or cSLO level of minute visualization. In experiments where gross morphological appearance of the retina is required, the fundus camera may still provide a significant tool. However, out of the various imaging modalities mentioned in this review, the fundus camera stands alone as the most inexpensive but also the most subjective means of assessment. The images produced are highly subjective to the viewer and can therefore potentially differ in diagnosis from person to person. Future directions such as automatic tagging of fundus imaging by using artificial intelligence algorithms (43) may yield a computerized tool assisting ophthalmologists and researchers, although the implementation of such tools in preclinical studies has to be determined. OCT, AO, and cSLO all provide images that can be objectively measured and are therefore considered more accurate and yield quantitative outcome measures that may be translated from animals to humans.

OCT is a time-proven platform that is recognized for being able to accurately evaluate different retinal cell layers in vivo. Measuring thickness of the retinal layers can be computerized to yield mathematical numbers that have been cross-checked as accurate by follow-up histological analysis. For experiments needing an accurate means of interpreting retinal layer health, OCT provides the most appropriate medium and can be viewed in a three-dimensional configuration. Future development of wide field OCT, as well as tools for automatic segmentation of retinal layers in rodent models and diseased eyes, will further improve the accuracy of this tool.

Recent questions have been raised for future directives as to whether or not OCTA can replace fundus angiography in diagnostic capability. One study compared these techniques longitudinally in diabetic retinopathic patients, concluding that OCTA cannot currently be used as a stand-alone method over fundus angiography (138). This segues into OCTA's main drawbacks, the lack of global consensus on segmentation and long learning curve needed to capture and interpret the images.

AO technology is the one imaging method described in this review that cannot function independently, which makes it both advantageous and disadvantageous to work with. First off, its ability in itself allows for incredibly clear visualization that has remained unmatched since its introduction to the market. Studies that seek to monitor and carefully examine the minutia of the retina down to single cells are those that stand to benefit the most from AO. Advantages of AO having a dependency on other imaging modalities is that they can be applied to and systematically improve image quality for any and all other preexisting platforms. Unfortunately, this advantage comes with a price as $\mathrm{AO}$ equipment is understandably still very expensive. On top of purchasing the $\mathrm{AO}$ equipment itself, the dependency of the equipment means that another system must be purchased and optimized to the equipment, further raising the price. Additionally, the equipment is exceedingly complex and time consuming to learn its basic operating system, so experiments that require multiple angles of data collection sets may find the process more time consuming.

Lastly, DARC with cSLO grants impressive power to those seeking to study RGC death in vivo. Longitudinal studies of glaucoma and PD appear to be the pathologies most commonly investigated with this equipment so far, yet the boundaries for retinopathies it can be applied to extend far further. The relative ease and reproducibility of the technique permits clear and straightforward interpretation of the results. In the future it would be exciting to see a development in this technology that would allow for fluorescently tagged RGCs to be automatically, instead of manually, counted. The main drawback that potentially limits DARC's widespread use is its unstandardized quantification in differentiating between pathological and physiological RGC death. Although the studies mentioned in this review maintain its accuracy as an early diagnostic method of retinal and central nervous system pathologies, care should be given when setting up future experiments to have a concrete control group so as to subtract any baseline fluorescence from the experimental group. Doing so would provide stronger sets of data collection.

Another promising direction in retinal imaging is its 
potential use for early detection of brain pathologies. With the retina being a "window to the brain", the retinal imaging technologies provide potentially earlier diagnostic capabilities and can concede the development of new regenerative interventions in retinal and brain neurodegeneration diseases.

\section{Acknowledgments}

Funding: None.

\section{Footnote}

Provenance and Peer Review: This article was commissioned by the Guest Editor (Susanna S. Park) for the series "Novel Tools and Therapies for Ocular Regeneration" published in Annals of Translational Medicine. The article was sent for external peer review organized by the Guest Editor and the editorial office.

Peer Review File: Available at http://dx.doi.org/10.21037/ atm-20-4355

Conflicts of Interest: All authors have completed the ICMJE uniform disclosure form (available at http://dx.doi. org/10.21037/atm-20-4355). The series "Novel Tools and Therapies for Ocular Regeneration" was commissioned by the editorial office without any funding or sponsorship. The authors have no other conflicts of interest to declare.

Ethical Statement: The authors are accountable for all aspects of the work in ensuring that questions related to the accuracy or integrity of any part of the work are appropriately investigated and resolved.

Open Access Statement: This is an Open Access article distributed in accordance with the Creative Commons Attribution-NonCommercial-NoDerivs 4.0 International License (CC BY-NC-ND 4.0), which permits the noncommercial replication and distribution of the article with the strict proviso that no changes or edits are made and the original work is properly cited (including links to both the formal publication through the relevant DOI and the license). See: https://creativecommons.org/licenses/by-nc-nd/4.0/.

\section{References}

1. FDA. FDA approves novel gene therapy to treat patients with a rare form of inherited vision loss. Available online: https://www.fda.gov/news-events/press-announcements/ fda-approves-novel-gene-therapy-treat-patients-rareform-inherited-vision-loss

2. Machida S, Kondo M, Jamison JA, et al. P23H rhodopsin transgenic rat: correlation of retinal function with histopathology. Invest Ophthalmol Vis Sci 2000;41:3200-9.

3. Tzameret A, Sher I, Belkin M, et al. Transplantation of human bone marrow mesenchymal stem cells as a thin subretinal layer ameliorates retinal degeneration in a rat model of retinal dystrophy. Exp Eye Res 2014;118:135-44.

4. Holekamp N, Wykoff CC, Schmitz-Valckenberg S, et al. Natural History of Geographic Atrophy Secondary to Age-Related Macular Degeneration: Results from the Prospective Proxima A and B Clinical Trials. Ophthalmology 2020;127:769-83.

5. Tee JJL, Yang Y, Kalitzeos A, et al. Natural History Study of Retinal Structure, Progression, and Symmetry Using Ellipzoid Zone Metrics in RPGR-Associated Retinopathy. Am J Ophthalmol 2019;198:111-23.

6. Rangaswamy NV, Patel HM, Locke KG, et al. A comparison of visual field sensitivity to photoreceptor thickness in retinitis pigmentosa. Invest Ophthalmol Vis Sci 2010;51:4213-9.

7. Gardiner SK, Demirel S, Reynaud J, et al. Changes in retinal nerve fiber layer reflectance intensity as a predictor of functional progression in glaucoma. Invest Ophthalmol Vis Sci 2016;57:1221-7.

8. Khani SC, Pawlyk BS, Bulgakov OV, et al. AAV-mediated expression targeting of rod and cone photoreceptors with a human rhodopsin kinase promoter. Invest Ophthalmol Vis Sci 2007;48:3954-61.

9. Li Q, Timmers AM, Guy J, et al. Cone-specific expression using a human red opsin promoter in recombinant AAV. Vision Res 2008;48:332-8.

10. Tzameret A, Sher I, Belkin M, et al. Epiretinal transplantation of human bone marrow mesenchymal stem cells rescues retinal and vision function in a rat model of retinal degeneration. Stem Cell Res 2015;15:387-94.

11. Georgiadis A, Tschernutter M, Bainbridge JWB, et al. AAV-mediated knockdown of peripherin-2 in vivo using miRNA-based hairpins. Gene Ther 2010;17:486-93.

12. LaVail MM, Yasumura D, Matthes MT, et al. Gene therapy for MERTK-associated retinal degenerations. Adv Exp Med Biol 2016;854:487-93.

13. McGill TJ, Prusky GT, Douglas RM, et al. Discordant anatomical, electrophysiological, and visual behavioral 
profiles of retinal degeneration in rat models of retinal degenerative disease. Invest Ophthalmol Vis Sci 2012;53:6232-44.

14. May CA, Horneber M, Lütjen-Drecoll E. Quantitative and morphological changes of the choroid vasculature in RCS rats and their congenic controls. Exp Eye Res 1996;63:75-84.

15. Huang YM, Yin ZQ, Liu K, et al. Temporal and spatial characteristics of cone degeneration in RCS rats. Jpn J Ophthalmol 2011;55:155-62.

16. LaVail MM, Pinto LH, Yasumura D. The interphotoreceptor matrix in rats with inherited retinal dystrophy. Invest Ophthalmol Vis Sci 1981;21:658-68.

17. Herron WL, Riegel BW, Myers OE, et al. Retinal dystrophy in the rat--a pigment epithelial disease. Invest Ophthalmol 1969;8:595-604.

18. Bourne MC, Campbell DA, Tansley K. Hereditary degeneration of the rat retina. Br J Ophthalmol 1938;22:613-23.

19. Vollrath D, Feng W, Duncan JL, et al. Correction of the retinal dystrophy phenotype of the RCS rat by viral gene transfer of Mertk. Proc Natl Acad Sci USA 2001;98:12584-9.

20. Tschernutter M, Schlichtenbrede FC, Howe S, et al. Long-term preservation of retinal function in the RCS rat model of retinitis pigmentosa following lentivirusmediated gene therapy. Gene Ther 2005;12:694-701.

21. Jacobson SG, Boye SL, Aleman TS, et al. Safety in nonhuman primates of ocular AAV2-RPE65, a candidate treatment for blindness in Leber congenital amaurosis. Hum Gene Ther 2006;17:845-58.

22. Duncan JL, LaVail MM, Yasumura D, et al. An RCS-like retinal dystrophy phenotype in mer knockout mice. Invest Ophthalmol Vis Sci 2003;44:826-38.

23. Ross JW, Fernandez de Castro JP, Zhao J, et al. Generation of an inbred miniature pig model of retinitis pigmentosa. Invest Ophthalmol Vis Sci 2012;53:501-7.

24. Patel TP, Kim TN, Yu G, et al. Smartphone-Based, Rapid, Wide-Field Fundus Photography for Diagnosis of Pediatric Retinal Diseases. Transl Vis Sci Technol 2019;8:29.

25. Kim TN, Myers F, Reber C, et al. A Smartphone-Based Tool for Rapid, Portable, and Automated Wide-Field Retinal Imaging. Transl Vis Sci Technol 2018;7:21.

26. Williams DR. Imaging single cells in the living retina. Vision Res 2011;51:1379-96.

27. Ahmad SS. An introduction to DARC technology. Saudi J Ophthalmol 2017;31:38-41.
28. McGregor JE, Godat T, Dhakal KR, et al. Optogenetic restoration of retinal ganglion cell activity in the living primate. Nat Commun 2020;11:1703.

29. Fujimoto JG, Pitris C, Boppart SA, et al. Optical coherence tomography: an emerging technology for biomedical imaging and optical biopsy. Neoplasia 2000;2:9-25.

30. Roy R, Saurabh K, Thomas NR, et al. Validation of multicolor imaging of diabetic retinopathy lesions vis a vis conventional color fundus photographs. Ophthalmic Surg Lasers Imaging Retina 2019;50:8-15.

31. Ballios BG, Weisbrod D, Kohly R, et al. Wide-field truecolour imaging and clinical characterization of a novel GRK1 mutation in Oguchi disease. Doc Ophthalmol 2020. [Epub ahead of print].

32. Cooper AE, Ahonen S, Rowlan JS, et al. A novel form of progressive retinal atrophy in Swedish vallhund dogs. PLoS One 2014;9:e106610.

33. Herron WL, Riegel BW, Brennan E, Rubin ML. Retinal dystrophy in the pigmented rat. Available online: https:// www.semanticscholar.org/paper/Retinal-dystrophy-in-thepigmented-rat.-Herron-Riegel/45f9097d7e27216fc449294 f263bc9a410b12bc5

34. Karan G, Lillo C, Yang Z, et al. Lipofuscin accumulation, abnormal electrophysiology, and photoreceptor degeneration in mutant ELOVL4 transgenic mice: a model for macular degeneration. Proc Natl Acad Sci USA 2005;102:4164-9.

35. Noel JM, Fernandez de Castro JP, Demarco PJ, et al. Iodoacetic acid, but not sodium iodate, creates an inducible swine model of photoreceptor damage. Exp Eye Res 2012;97:137-47.

36. Komáromy AM, Alexander JJ, Cooper AE, et al. Targeting gene expression to cones with human cone opsin promoters in recombinant AAV. Gene Ther 2008;15:1049-55.

37. Weed L, Ammar MJ, Zhou S, et al. Safety of SameEye Subretinal Sequential Readministration of AAV2hRPE65v2 in Non-human Primates. Mol Ther Methods Clin Dev 2019;15:133-48.

38. Krohne TU, Westenskow PD, Kurihara T, et al. Generation of retinal pigment epithelial cells from small molecules and OCT4 reprogrammed human induced pluripotent stem cells. Stem Cells Transl Med 2012;1:96-109.

39. Yang Y, Mohand-Said S, Léveillard T, et al. Transplantation of photoreceptor and total neural retina preserves cone function in $\mathrm{P} 23 \mathrm{H}$ rhodopsin transgenic rat. PLoS One 2010;5:e13469. 
40. da Cruz L, Fynes K, Georgiadis O, et al. Phase 1 clinical study of an embryonic stem cell-derived retinal pigment epithelium patch in age-related macular degeneration. Nat Biotechnol 2018;36:328-37.

41. Conlon TJ, Deng WT, Erger K, et al. Preclinical potency and safety studies of an AAV2-mediated gene therapy vector for the treatment of MERTK associated retinitis pigmentosa. Hum Gene Ther Clin Dev 2013;24:23-8.

42. Li S, Datta S, Brabbit E, et al. Nr2e3 is a genetic modifier that rescues retinal degeneration and promotes homeostasis in multiple models of retinitis pigmentosa. Gene Ther 2020. [Epub ahead of print].

43. Lim G, Bellemo V, Xie Y, et al. Different fundus imaging modalities and technical factors in AI screening for diabetic retinopathy: a review. Eye Vis (Lond) 2020;7:21.

44. Piccolino FC, Borgia L, Zinicola E, et al. Pre-injection fluorescence in indocyanine green angiography. Ophthalmology 1996;103:1837-45.

45. Elsner AE, Burns SA, Weiter JJ, et al. Infrared imaging of sub-retinal structures in the human ocular fundus. Vision Res 1996;36:191-205.

46. Keilhauer CN, Delori FC. Near-infrared autofluorescence imaging of the fundus: visualization of ocular melanin. Invest Ophthalmol Vis Sci 2006;47:3556-64.

47. Ly A, Nivison-Smith L, Assaad N, et al. Infrared reflectance imaging in age-related macular degeneration. Ophthalmic Physiol Opt 2016;36:303-16.

48. Rotenstreich Y, Tzameret A, Kalish SE, et al. A minimally invasive adjustable-depth blunt injector for delivery of pharmaceuticals into the posterior pole. Acta Ophthalmol 2017;95:e197-205.

49. von Rückmann A, Fitzke FW, Bird AC. Distribution of fundus autofluorescence with a scanning laser ophthalmoscope. Br J Ophthalmol 1995;79:407-12.

50. Sparrow JR, Duncker T. Fundus Autofluorescence and RPE Lipofuscin in Age-Related Macular Degeneration. J Clin Med 2014;3:1302-21.

51. Sparrow JR, Hicks D, Hamel CP. The retinal pigment epithelium in health and disease. Curr Mol Med 2010;10:802-23.

52. Sparrow JR, Yoon KD, Wu Y, et al. Interpretations of fundus autofluorescence from studies of the bisretinoids of the retina. Invest Ophthalmol Vis Sci 2010;51:4351-7.

53. Sparrow JR, Wu Y, Nagasaki T, et al. Fundus autofluorescence and the bisretinoids of retina. Photochem Photobiol Sci 2010;9:1480-9.

54. Delori FC, Goger DG, Dorey CK. Age-related accumulation and spatial distribution of lipofuscin in
RPE of normal subjects. Invest Ophthalmol Vis Sci 2001;42:1855-66.

55. Bubis E, Sher I, Skaat A, et al. Blue autofluorescence fundus imaging for monitoring retinal degeneration in royal college of surgeons rats. Transl Vis Sci Technol 2019;8:26

56. Schmitz-Valckenberg S, Jorzik J, Unnebrink K, et al. Analysis of digital scanning laser ophthalmoscopy fundus autofluorescence images of geographic atrophy in advanced age-related macular degeneration. Graefes Arch Clin Exp Ophthalmol 2002;240:73-8.

57. Fleckenstein M, Charbel Issa P, Helb HM, et al. Highresolution spectral domain-OCT imaging in geographic atrophy associated with age-related macular degeneration. Invest Ophthalmol Vis Sci 2008;49:4137-44.

58. Panthier C, Querques G, Puche N, et al. Evaluation of semiautomated measurement of geographic atrophy in age-related macular degeneration by fundus autofluorescence in clinical setting. Retina (Philadelphia, Pa) 2014;34:576-82.

59. Miere A, Capuano V, Serra R, et al. Evaluation of patchy atrophy secondary to high myopia by semiautomated software for fundus autofluorescence analysis. Retina (Philadelphia, Pa) 2018;38:1301-6.

60. Groh J, Stadler D, Buttmann M, et al. Non-invasive assessment of retinal alterations in mouse models of infantile and juvenile neuronal ceroid lipofuscinosis by spectral domain optical coherence tomography. Acta Neuropathol Commun 2014;2:54.

61. Moisseiev E, Smit-McBride Z, Oltjen S, et al. Intravitreal administration of human bone marrow CD34+ stem cells in a murine model of retinal degeneration. Invest Ophthalmol Vis Sci 2016;57:4125-35.

62. Wassmer SJ, Carvalho LS, György B, et al. Exosomeassociated AAV2 vector mediates robust gene delivery into the murine retina upon intravitreal injection. Sci Rep 2017;7:45329.

63. Manivannan A, Sharp PF, Phillips RP, et al. Digital fundus imaging using a scanning laser ophthalmoscope. Physiol Meas 1993;14:43-56.

64. Huber G, Beck SC, Grimm C, et al. Spectral domain optical coherence tomography in mouse models of retinal degeneration. Invest Ophthalmol Vis Sci 2009;50:5888-95.

65. Pinilla I, Fernández-Sánchez L, Segura FJ, et al. Long time remodeling during retinal degeneration evaluated by optical coherence tomography, immunocytochemistry and fundus autofluorescence. Exp Eye Res 2016;150:122-34.

66. Yang Y, Ng TK, Ye C, et al. Assessing sodium iodate- 
induced outer retinal changes in rats using confocal scanning laser ophthalmoscopy and optical coherence tomography. Invest Ophthalmol Vis Sci 2014;55:1696-705.

67. Drexler W, Fujimoto JG. State-of-the-art retinal optical coherence tomography. Prog Retin Eye Res 2008;27:45-88.

68. Keane PA, Sadda SR. Retinal imaging in the twentyfirst century: state of the art and future directions. Ophthalmology 2014;121:2489-500.

69. Virgili G, Menchini F, Casazza G, et al. Optical coherence tomography (OCT) for detection of macular oedema in patients with diabetic retinopathy. Cochrane Database Syst Rev 2015;1:CD008081.

70. Lee KM, Lee EJ, Kim TW, et al. Comparison of the Abilities of SD-OCT and SS-OCT in Evaluating the Thickness of the Macular Inner Retinal Layer for Glaucoma Diagnosis. PLoS One 2016;11:e0147964.

71. Silverman AL, Hammel N, Khachatryan N, et al. Diagnostic Accuracy of the Spectralis and Cirrus Reference Databases in Differentiating between Healthy and Early Glaucoma Eyes. Ophthalmology 2016;123:408-14.

72. Medeiros FA, Zangwill LM, Bowd C, et al. The structure and function relationship in glaucoma: implications for detection of progression and measurement of rates of change. Invest Ophthalmol Vis Sci 2012;53:6939-46.

73. Allingham MJ, Tserentsoodol N, Saloupis P, et al. Aldosterone Exposure Causes Increased Retinal Edema and Severe Retinopathy Following Laser-Induced Retinal Vein Occlusion in Mice. Invest Ophthalmol Vis Sci 2018;59:3355-65.

74. Ryals RC, Andrews MD, Datta S, et al. Long-term Characterization of Retinal Degeneration in Royal College of Surgeons Rats Using Spectral-Domain Optical Coherence Tomography. Invest Ophthalmol Vis Sci 2017;58:1378-86.

75. Adachi K, Takahashi S, Yamauchi K, et al. Optical Coherence Tomography of Retinal Degeneration in Royal College of Surgeons Rats and Its Correlation with Morphology and Electroretinography. PLoS One 2016;11:e0162835.

76. Cuenca N, Fernández-Sánchez L, Sauvé Y, et al. Correlation between SD-OCT, immunocytochemistry and functional findings in an animal model of retinal degeneration. Front Neuroanat 2014;8:151.

77. Orhan E, Dalkara D, Neuillé M, et al. Genotypic and phenotypic characterization of $\mathrm{P} 23 \mathrm{H}$ line 1 rat model. PLoS One 2015;10:e0127319.

78. Nakazawa M, Hara A, Ishiguro SI. Optical coherence tomography of animal models of retinitis pigmentosa: from animal studies to clinical applications. Biomed Res Int 2019;2019:8276140.

79. Monai N, Yamauchi K, Tanabu R, et al. Characterization of photoreceptor degeneration in the rhodopsin $\mathrm{P} 23 \mathrm{H}$ transgenic rat line 2 using optical coherence tomography. PLoS One 2018;13:e0193778.

80. Fischer MD, Huber G, Beck SC, et al. Noninvasive, in vivo assessment of mouse retinal structure using optical coherence tomography. PLoS One 2009;4:e7507.

81. Hasegawa T, Ikeda HO, Nakano N, et al. Changes in morphology and visual function over time in mouse models of retinal degeneration: an SD-OCT, histology, and electroretinography study. Jpn J Ophthalmol 2016;60:111-25.

82. Muraoka Y, Ikeda HO, Nakano N, et al. Real-time imaging of rabbit retina with retinal degeneration by using spectral-domain optical coherence tomography. PLoS One 2012;7:e36135.

83. Nakagami Y, Hatano E, Inoue T, et al. Cytoprotective effects of a novel nrf2 activator, RS9, in rhodopsin pro347leu rabbits. Curr Eye Res 2016;41:1123-6.

84. Shirai H, Mandai M, Matsushita K, et al. Transplantation of human embryonic stem cell-derived retinal tissue in two primate models of retinal degeneration. Proc Natl Acad Sci USA 2016;113:E81-90.

85. Zhi Z, Chao JR, Wietecha T, et al. Noninvasive imaging of retinal morphology and microvasculature in obese mice using optical coherence tomography and optical microangiography. Invest Ophthalmol Vis Sci 2014;55:1024-30.

86. Tawfik A, Mohamed R, Elsherbiny NM, et al. Homocysteine: A potential biomarker for diabetic retinopathy. J Clin Med 2019;8:121.

87. Rovere G, Nadal-Nicolás FM, Agudo-Barriuso M, et al. Comparison of retinal nerve fiber layer thinning and retinal ganglion cell loss after optic nerve transection in adult albino rats. Invest Ophthalmol Vis Sci 2015;56:4487-98.

88. Tu HY, Watanabe T, Shirai H, et al. Medium- to longterm survival and functional examination of human iPSC-derived retinas in rat and primate models of retinal degeneration. EBioMedicine 2019;39:562-74.

89. Lin B, McLelland BT, Mathur A, et al. Sheets of human retinal progenitor transplants improve vision in rats with severe retinal degeneration. Exp Eye Res 2018;174:13-28.

90. Zhou XM, Sun J, Yuan H, et al. A rat model for studying neural stem cell transplantation. Acta Pharmacol Sin 2009;30:1496-504. 
91. Lorach H, Kang S, Bhuckory MB, et al. Transplantation of mature photoreceptors in rodents with retinal degeneration. Transl Vis Sci Technol 2019;8:30.

92. Lorach H, Kang S, Dalal R, et al. Long-term Rescue of Photoreceptors in a Rodent Model of Retinitis Pigmentosa Associated with MERTK Mutation. Sci Rep 2018;8:11312.

93. Zeng Y, Petralia RS, Vijayasarathy C, et al. Retinal Structure and Gene Therapy Outcome in RetinoschisinDeficient Mice Assessed by Spectral-Domain Optical Coherence Tomography. Invest Ophthalmol Vis Sci 2016;57:OCT277-87.

94. Mackay DS, Henderson RH, Sergouniotis PI, et al. Novel mutations in MERTK associated with childhood onset rod-cone dystrophy. Mol Vis 2010;16:369-77.

95. Charbel Issa P, Bolz HJ, Ebermann I, et al. Characterisation of severe rod-cone dystrophy in a consanguineous family with a splice site mutation in the MERTK gene. Br J Ophthalmol 2009;93:920-5.

96. Sher I, Tzameret A, Szalapak AM, et al. Multimodal assessment of corneal erosions using optical coherence tomography and automated grading of fluorescein staining in a rabbit dry eye model. Transl Vis Sci Technol 2019;8:27.

97. Sher I, Tzameret A, Goldberg Z, et al. Repetitive magnetic stimulation protects corneal epithelium in a rabbit model of short-term exposure keratopathy. Ocul Surf 2020;18:64-73.

98. Srinivasan PP, Heflin SJ, Izatt JA, et al. Automatic segmentation of up to ten layer boundaries in SD-OCT images of the mouse retina with and without missing layers due to pathology. Biomed Opt Express 2014;5:348-65.

99. McNabb RP, Polans J, Keller B, et al. Wide-field whole eye OCT system with demonstration of quantitative retinal curvature estimation. Biomed Opt Express 2018;10:338-55.

100.Leitgeb RA. En face optical coherence tomography: a technology review [Invited]. Biomed Opt Express 2019;10:2177-201.

101.Hartong DT, Berson EL, Dryja TP. Retinitis pigmentosa. Lancet 2006;368:1795-809.

102. Makita S, Hong Y, Yamanari M, et al. Optical coherence angiography. Opt Express 2006;14:7821.

103. Meyer JH, Larsen PP, Strack C, et al. Optical coherence tomography angiography (OCT-A) in an animal model of laser-induced choroidal neovascularization. Exp Eye Res 2019;184:162-71.

104. Kim TH, Son T, Le D, et al. Longitudinal OCT and OCTA monitoring reveals accelerated regression of hyaloid vessels in retinal degeneration $10(\operatorname{rd} 10)$ mice. Sci Rep 2019;9:16685.

105. Cuenca N, Ortuño-Lizarán I, Sánchez-Sáez X, et al. Interpretation of OCT and OCTA images from a histological approach: Clinical and experimental implications. Prog Retin Eye Res 2020. [Epub ahead of print].

106. Spaide RF, Klancnik JM, Cooney MJ. Retinal vascular layers imaged by fluorescein angiography and optical coherence tomography angiography. JAMA Ophthalmol 2015;133:45-50.

107. Koustenis A, Harris A, Gross J, et al. Optical coherence tomography angiography: an overview of the technology and an assessment of applications for clinical research. Br J Ophthalmol 2017;101:16-20.

108. Balaratnasingam C, An D, Sakurada Y, et al. Comparisons Between Histology and Optical Coherence Tomography Angiography of the Periarterial Capillary-Free Zone. Am J Ophthalmol 2018;189:55-64.

109. Spaide RF, Fujimoto JG, Waheed NK, et al. Optical coherence tomography angiography. Prog Retin Eye Res 2018;64:1-55.

110.De Oliveira PRC, Berger AR, Chow DR. Optical coherence tomography angiography in chorioretinal disorders. Can J Ophthalmol 2017;52:125-36.

111. Spaide RF, Fujimoto JG, Waheed NK. Image artifacts in optical coherence tomography angiography. Retina (Philadelphia, Pa) 2015;35:2163-80.

112. Chen FK, Viljoen RD, Bukowska DM. Classification of image artefacts in optical coherence tomography angiography of the choroid in macular diseases. Clin Experiment Ophthalmol 2016;44:388-99.

113. Kashani AH, Chen CL, Gahm JK, et al. Optical coherence tomography angiography: A comprehensive review of current methods and clinical applications. Prog Retin Eye Res 2017;60:66-100.

114.Park JR, Choi W, Hong HK, et al. Imaging Laser-Induced Choroidal Neovascularization in the Rodent Retina Using Optical Coherence Tomography Angiography. Invest Ophthalmol Vis Sci 2016;57:OCT331-40.

115. Kim TH, Son T, Lu Y, et al. Comparative Optical Coherence Tomography Angiography of Wild-Type and rd10 Mouse Retinas. Transl Vis Sci Technol 2018;7:42.

116. Moisseiev E, Anderson JD, Oltjen S, et al. Protective Effect of Intravitreal Administration of Exosomes Derived from Mesenchymal Stem Cells on Retinal Ischemia. Curr Eye Res 2017;42:1358-67.

117.Lombardo M, Serrao S, Devaney N, et al. Adaptive optics 
technology for high-resolution retinal imaging. Sensors (Basel) 2012;13:334-66.

118. Godara P, Dubis AM, Roorda A, et al. Adaptive optics retinal imaging: emerging clinical applications. Optom Vis Sci 2010;87:930-41.

119. Salter PS, Booth MJ. Adaptive optics in laser processing. Light Sci Appl 2019;8:110.

120. Burns SA, Elsner AE, Sapoznik KA, et al. Adaptive optics imaging of the human retina. Prog Retin Eye Res 2019;68:1-30.

121. Harmening WM, Sincich LC. Adaptive Optics for Photoreceptor-Targeted Psychophysics. In: Bille JF, editor. High resolution imaging in microscopy and ophthalmology: new frontiers in biomedical optics. Cham (CH): Springer, 2019.

122.Jian Y, Zawadzki RJ, Sarunic MV. Adaptive optics optical coherence tomography for in vivo mouse retinal imaging. J Biomed Opt 2013;18:56007.

123. Heisler M, Ju MJ, Bhalla M, et al. Automated identification of cone photoreceptors in adaptive optics optical coherence tomography images using transfer learning. Biomed Opt Express 2018;9:5353-67.

124. Reumueller A, Schmidt-Erfurth U, Salas M, et al. ThreeDimensional Adaptive Optics-Assisted Visualization of Photoreceptors in Healthy and Pathologically Aged Eyes. Invest Ophthalmol Vis Sci 2019;60:1144-55.

125.Potic J, Bergin C, Giacuzzo C, et al. Changes in visual acuity and photoreceptor density using adaptive optics after retinal detachment repair. Retina 2018. [Epub ahead of print].

126. Wang X, Zhao L, Zhang J, et al. Requirement for microglia for the maintenance of synaptic function and integrity in the mature retina. J Neurosci 2016;36:2827-42.

127. Muthiah MN, Keane PA, Zhong J, et al. Adaptive optics imaging shows rescue of macula cone photoreceptors. Ophthalmology 2014;121:430-1.e3.

128. Miller EB, Zhang P, Ching K, et al. In vivo imaging reveals transient microglia recruitment and functional recovery of photoreceptor signaling after injury. Proc Natl Acad Sci USA 2019;116:16603-12.

129. Marcos S, Werner JS, Burns SA, et al. Vision science and adaptive optics, the state of the field. Vision Res 2017;132:3-33.

130. Normando EM, Turner LA, Cordeiro MF. The potential of annexin-labelling for the diagnosis and follow-up of glaucoma. Cell Tissue Res 2013;353:279-85.

131.Diekmann H, Fischer D. Glaucoma and optic nerve repair. Cell Tissue Res 2013;353:327-37.

132. Tham YC, Li X, Wong TY, et al. Global prevalence of glaucoma and projections of glaucoma burden through 2040: a systematic review and meta-analysis. Ophthalmology 2014;121:2081-90.

133. Yap TE, Shamsher E, Guo L, et al. Ophthalmic research lecture 2018: DARC as a potential surrogate marker. Ophthalmic Res 2020;63:1-7.

134. Davis BM, Tian K, Pahlitzsch M, et al. Topical Coenzyme Q10 demonstrates mitochondrial-mediated neuroprotection in a rodent model of ocular hypertension. Mitochondrion 2017;36:114-23.

135. Normando EM, Davis BM, De Groef L, et al. The retina as an early biomarker of neurodegeneration in a rotenoneinduced model of Parkinson's disease: evidence for a neuroprotective effect of rosiglitazone in the eye and brain. Acta Neuropathol Commun 2016;4:86.

136. Cordeiro MF, Migdal C, Bloom P, et al. Imaging apoptosis in the eye. Eye (Lond) 2011;25:545-53.

137.Pahlitzsch M. Potential impact of DARC technology in neuroprotective therapies. Klin Monbl Augenheilkd 2020;237:140-2.

138. Russell JF, Shi Y, Hinkle JW, et al. Longitudinal Wide-Field Swept-Source OCT Angiography of Neovascularization in Proliferative Diabetic Retinopathy after Panretinal Photocoagulation. Ophthalmol Retina 2019;3:350-61.
Cite this article as: Sher I, Moverman D, Ketter-Katz H, Moisseiev E, Rotenstreich Y. In vivo retinal imaging in translational regenerative research. Ann Transl Med 2020;8(17):1096. doi: 10.21037/atm-20-4355 